\title{
Neurotización: concepto clave en reparación nerviosa
}

\section{Neurotization: Key Concept in Nerve Repair}

Recibido: 11 Marzo 2021 | Aceptado: 27 Abril 2021

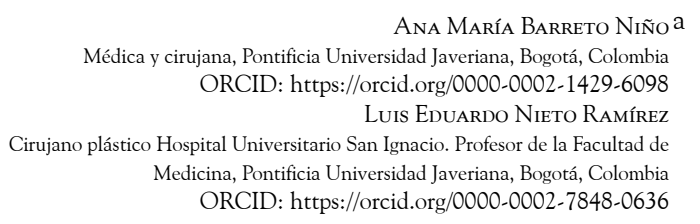

\section{RESUMEN}

La neurotización es el fenómeno por medio del cual un brote axonal alcanza la estructura de un nervio periférico lesionado, coloniza el órgano o tejido blanco de dicho nervio y produce en este la función que tiene determinado el cuerpo celular al cual pertenece esta nueva prolongación axonal. Los tipos de neurotización dependen del tejido que se va a reinervar. Teóricamente, cualquier tejido podría ser reinervado o neurotizado. Este artículo de revisión tiene el objetivo de explicar el fenómeno de neurotización y los diferentes tipos que hasta el momento han tenido aplicación clínica. Conocerlo es importante para indicar su aplicación en diferentes técnicas quirúrgicas y lograr cada día mejores resultados en pacientes con lesiones del nervio periférico, por ejemplo, la recuperación de lesiones tanto sensitivas como motoras, lesiones producidas por objetos cortantes, aplastamientos, quemaduras, heridas por arma de fuego o síndromes de compresión crónicos.

\section{Palabras clave}

neurotización; neurotización nerviosa; neurotización directa; neurotización de seno; neurotización de colgajos; neurotización corneal.

a Autora de correspondencia: barreto.ana@javeriana.edu.co

Cómo citar: Barreto Niño AM, Nieto LE. Neurotización: concepto clave en reparación nerviosa. Univ. Med. 2021;62(3). https://doi.org/10.1 1144/Javeriana.umed62-3.nccr

\begin{abstract}
Neurotization is the phenomenon by which an axonal sprout reaches the structure of an injured peripheral nerve, colonizes the organ or target tissue and produces in it, the function determined by the cell body to which this new axonal prolongation belongs. The types of neurotization depend on the tissue to be reinnervated, theoretically any tissue could be reinnervated or neurotized. This review article aims to explain the neurotization phenomenon and the different types that have had clinical application up to now, its knowledge being important to indicate its application in different surgical techniques and to achieve better results every day in patients with peripheral nerve injuries, achieving recovery from both sensory and motor injuries, injuries caused by sharp objects, crushing, burns, gunshot wounds or chronic compression syndromes.

Keywords

neurotization; nerve neurotization; direct neurotization; breast neurotization; flap neurotization; corneal neurotization.
\end{abstract}




\section{Introducción}

Las lesiones de nervios periféricos son altamente discapacitantes, ya sea por una pérdida funcional o por dolor crónico (1), para lo cual se han descrito múltiples técnicas quirúrgicas. En 1876, Albert E. describió el primer injerto nervioso y, en 1903, Harris y Low realizaron por primera vez una transferencia nerviosa. Con la evolución de estos procedimientos y el mejor entendimiento de las técnicas de reparación y la fisiopatología de la lesión nerviosa se obtienen cada vez mejores resultados en el diagnóstico y tratamiento de este tipo de lesiones.

La neurotización es el fenómeno biológico por medio del cual un brote axonal alcanza la estructura de un nervio periférico lesionado, coloniza su órgano o tejido blanco y produce en este la función que tiene determinado el cuerpo celular al que pertenece esta prolongación axonal. Así, cualquier tejido podría ser neurotizado y los tipos de neurotización dependen del tejido que se vaya a inervar (2).

Cuando se lesiona un nervio periférico, se producen una serie de fenómenos celulares y moleculares en cada una de las neuronas que lo conforman. Ello lleva nuevos axones al tejido que inerva dicho nervio y se logra así la recuperación de las funciones perdidas. Los cuerpos celulares aumentan considerablemente su actividad metabólica; a la vez, los axones proximales al sitio de lesión, seccionados o severamente afectados, incrementan el transporte de moléculas y factores, como preparación para su nuevo crecimiento desde el nodo de Ranvier más cercano (3). Al mismo tiempo, en los segmentos axonales distales ocurre el fenómeno de degeneración walleriana, el cual prepara los endoneuros para recibir los axones que van a crecer desde los cabos proximales. Cuando los axones alcanzan los tubos endoneurales del cabo distal, continúan su crecimiento con una progresión de aproximadamente $1 \mathrm{~mm} /$ día, hasta llegar al tejido $\mathrm{u}$ órgano efector donde se encuentran las placas neuromotoras o los receptores sensitivos. De esta manera, se recuperan las funciones determinadas de cada nervio (4).
Para que estos eventos ocurran de manera adecuada y haya recuperación funcional, se deben tener en cuenta varios factores, entre otros, el paso en el sitio de lesión de la mayor cantidad de axones del cabo proximal al cabo distal del nervio, lo cual ocurre con mayor efectividad en la medida en que dichos cabos estén más próximos y el esfuerzo celular y molecular sea el menor posible; tejidos sanos alrededor del nervio que se está regenerando; una adecuada técnica quirúrgica, y condiciones clínicas del propio paciente. Por eso, el diagnóstico temprano de la lesión y su reparación adecuada permite una excelente recuperación en todos los casos.

Para que esta neurotización ocurra se aplican diferentes técnicas quirúrgicas que buscan facilitar el crecimiento axonal en el nervio o tejido afectado, para que se recuperen las funciones sensitivas o motoras, propias de cada nervio.

\section{Neurotización nerviosa}

La neurotización nerviosa puede realizarse mediante neurorrafia primaria, injerto nervioso o transferencia nerviosa. La forma más sencilla y efectiva para reparar un nervio lesionado es la neurorrafia primaria (figura 1), que consiste en la coaptación directa de los cabos de la lesión. Ello permite que los axones alcancen su tejido efector, recuperen su función y completen la reinervación o neurotización primaria (5). 


\section{Figura 1}

Tipos de neurorrafia primaria: a) lesión nerviosa, b) neurorrafia epineural, c) neurorrafia perineural (interfascicular)

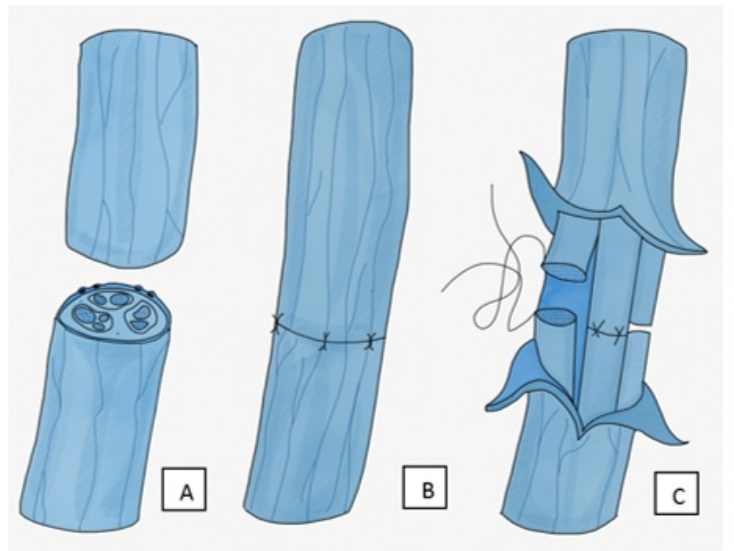

En los casos en los cuales no es posible dicha coaptación de los cabos de sección, se deben realizar injertos nerviosos (figura 2) o colocar tubos regenerativos (figura 3), construidos con materiales biológicos o sintéticos, para el paso de los axones al cabo distal, y así la neurotización del órgano efector, músculo (reneurotización o reinervación muscular) o piel (reneurotización o reinervación cutánea) correspondiente (6).

\section{Figura 2.}

Injerto de nervio interfascicular. Se utilizan segmentos de nervio donante para llenar el espacio en el nervio lesionado

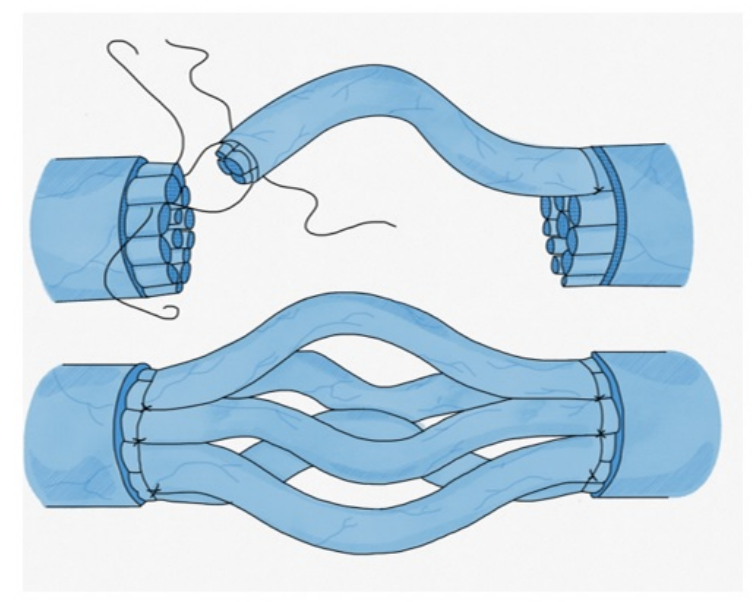

Figura 3.

Tubos regenerativos que guían el crecimiento axonal hasta el cabo distal. Se han diseñado de diversos materiales biológicos y sinteticos. Útiles para defectos de hasta $3 \mathrm{~cm}$

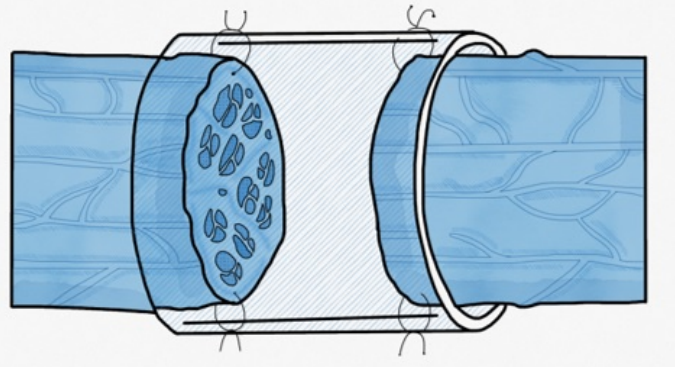

En casos de lesiones con tiempo de evolución mayor a 12 meses, en los que hay alta probabilidad de atrofia de la placa neuromotora o de los receptores sensitivos de la piel, en avulsiones de la raíz nerviosa de la médula espinal, lesiones proximales en las extremidades, lesiones severas con defectos de gran longitud (7) o abundante tejido cicatrizal que impida la adecuada regeneración axonal (8) se utilizan las transferencias nerviosas. Estas consisten en escoger un nervio donante sano, el cual se secciona en su totalidad o uno de sus fascículos, y transferir el cabo proximal de dicha sección hacia el cabo distal del nervio lesionado o receptor $(9,10)$. Aunque se sacrifica parcialmente la función del nervio donante (11), se transforma una lesión proximal en una lesión distal más cerca del órgano efector, para reducir el tiempo de reinervación o neurotización (12). Dicha coaptación nerviosa se puede realizar mediante neurorrafias término-terminales (13), términolaterales (14-16) (figura 4) o injertos nerviosos $(17,18)$ (figura 5). 


\section{Figura 4}

Diferentes formas de realizar anastomosis términolaterales. a) Epineuro intacto. b) Ventana de epineuro. c) Neurectomía parcial

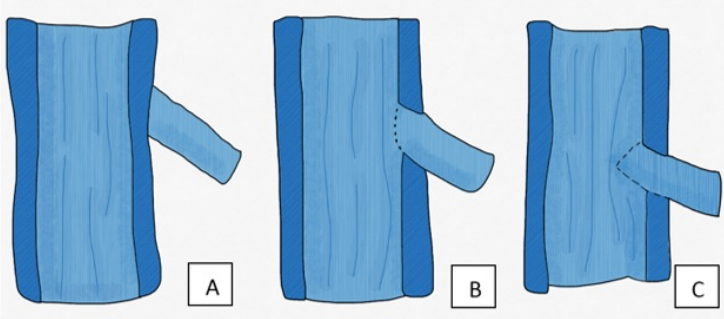

Figura 5.

Injerto de nervio colocado entre ramas del nervio facial sano a ramas del nervio facial comprometido, con el fin de suministrar axones sanos al nervio facial lesionado

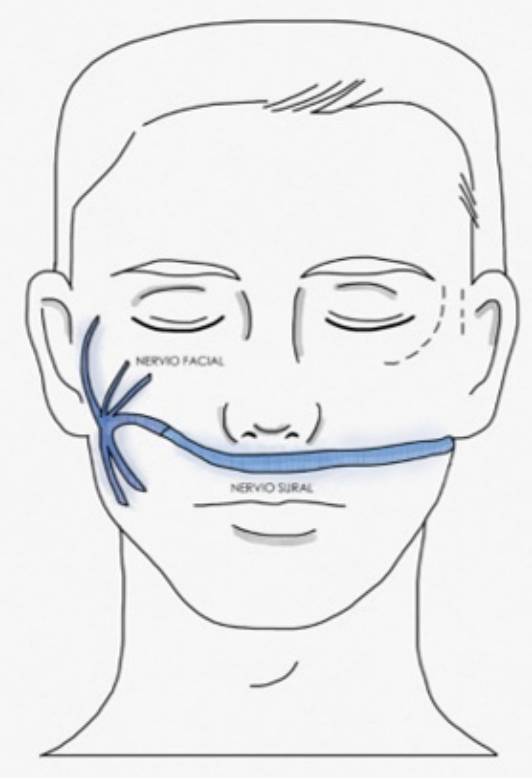

En lesiones tempranas del plexo braquial, las transferencias nerviosas son la mejor opción de tratamiento para lograr la neurotización y recuperación funcional de los grupos musculares y los dermatomas afectados (19). Dependiendo de cada tipo de lesión, se pueden realizar transferencias con nervios del mismo plexo braquial (neurotizaciones intraplexo) (20) o con nervios extraplexo (21), como el nervio accesorio del espinal, el nervio frénico o los nervios intercostales (22).

Una variante de neurotización muscular se realiza principalmente en parálisis facial, al utilizar un nervio donante, como el hipogloso o el nervio del músculo masetero (23), para dar axones que mantengan la placa neuromotora y los músculos faciales viables, mientras llegan los axones desde el lado contralateral sano por medio de un injerto de nervio sural y reinervan completamente los músculos de la hemicara afectada. A este tipo de procedimientos se les denomina baby sitter, al hacer referencia que "cuidan" los músculos de la atrofia mientras llega la neurotización definitiva (24).

\section{Neurotización muscular directa}

En casos en los cuales la reparación directa o la interposición de un injerto nervioso en una lesión de un nervio motor no es posible, debido a la pérdida del segmento distal del nervio lesionado o por destrucción de la placa neuromotora de uno o más grupos músculos (25), se puede implantar el segmento proximal del nervio lesionado directamente en el músculo (26), el cual debe tener un volumen satisfactorio, adecuada vascularidad y movilidad de las articulaciones donde él interviene (27), para que se logre una buena recuperación (figura 6).

\section{Figura 6}

Neurotización muscular directa inserción de cabo distal de un nervio sano en un músculo denervado

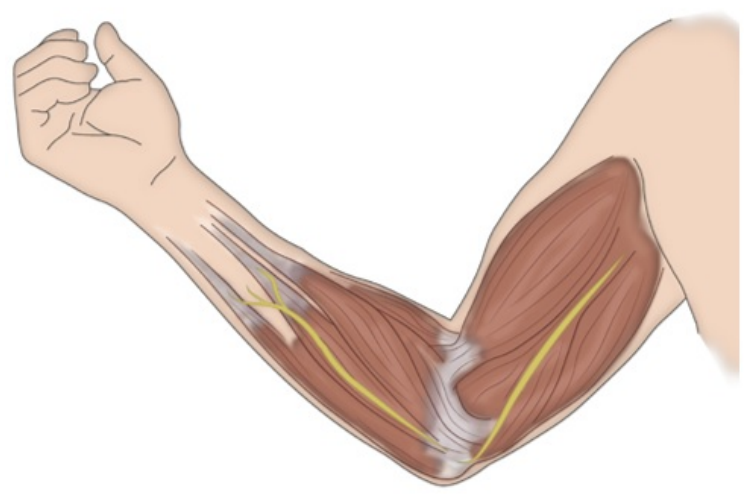


Los primeros estudios experimentales fueron realizados en 1908 por Von Hacker, quien implanto\# directamente la raíz espinal del nervio accesorio sobre un músculo trapecio paralizado (28). Siguieron este tipo de procedimientos, Heineke (29), Elsberg (30) y Steindler (31), a principios del siglo XX. En 1950, Hoffman demostró nuevas unidades motoras con estudios de electrodiagnóstico en músculos neurotizados directamente (32). En 1981, Brunelli y Monini publican resultados de casos clínicos donde se reimplantaron los nervios avulsionados dentro del músculo (33). Ello demostró que los músculos denervados son sensibles a la acetilcolina secretada por el nervio implantado $(34,35)$ y producen una adaptación funcional entre el nervio y la fibra muscular, al punto de constituir así una nueva placa neuromotora $(36,37)$.

\section{Neurotización músculo-nervio-músculo}

Esta forma de neurotización fue descrita por Millesi y Walzer, en 1985 (38), quienes interpusieron un injerto de nervio entre un músculo denervado y un músculo normal, para que brotes axonales del músculo normal crecieran por el injerto nervioso y neurotizaran fibras del músculo afectado (figura 7). Esta técnica se utiliza para reanimación facial selectiva (39).

\section{Figura 7}

Neurotización músculonerviomúsculo Colocación de un injerto nervioso que sirva de puente para que brotes axonales de un músculo sano lleguen al músculo denervado

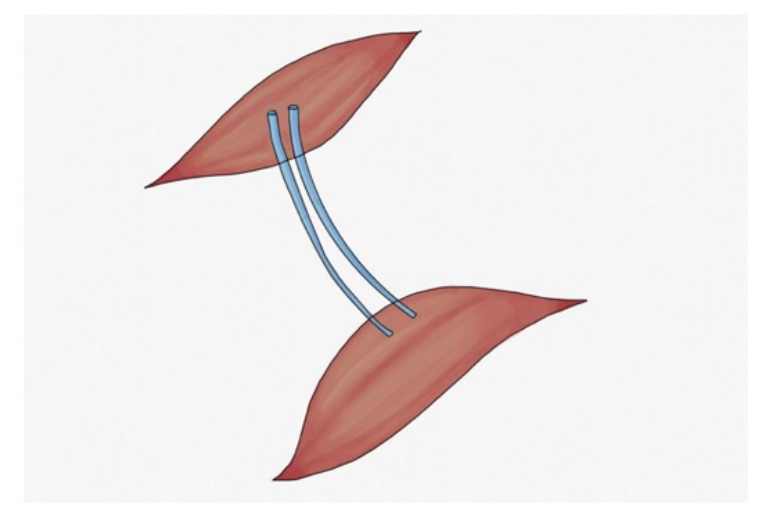

Millesi comunicó reinervación satisfactoria del músculo orbicular de la boca en 6 pacientes mediante la realización de un injerto músculonervio-músculo desde el músculo orbicular de la boca inervado hasta el músculo orbicular de la boca denervado contralateral (40).

Al realizar la disección en el músculo para implantar el injerto nervioso, la lesión de las fibras musculares causa una respuesta regenerativa de las fibras nerviosas presentes y lleva a estos brotes axonales a crecer a lo largo del injerto nervioso que ha sufrido degeneración walleriana, hasta fibras musculares del músculo denervado (41). Se ven mejores resultados entre músculos cercanos y sinérgicos (42). Esta técnica se ha utilizado en casos de denervación del músculo orbicularis oris (43), lesiones de la rama marginal del nervio facial y denervaciones laríngeas (44).

\section{Neurotización cutánea directa}

Cuando se restaura la sensibilidad de un colgajo cutáneo, ya sea desde los tejidos adyacentes luego de su integración a estos o ya sea por medio de una neurorrafia de un nervio cutáneo incluido en el colgajo con un nervio sensitivo del área receptora, se habla de neurotización cutánea directa (45). 
En reconstrucción mamaria (figura 8), de mano y región plantar (46), lo ideal es realizar este tipo de neurotizaciones con el fin de obtener algún grado de recuperación sensitiva erógena (47), sensibilidad táctil fina y protectiva, respectivamente.

\section{Figura 8}

Neurotización cutánea en reconstrucción de seno al realizar neurorrafia entre un nervio cutáneo incluido en el colgajo transferido y un nervio receptor intercostal

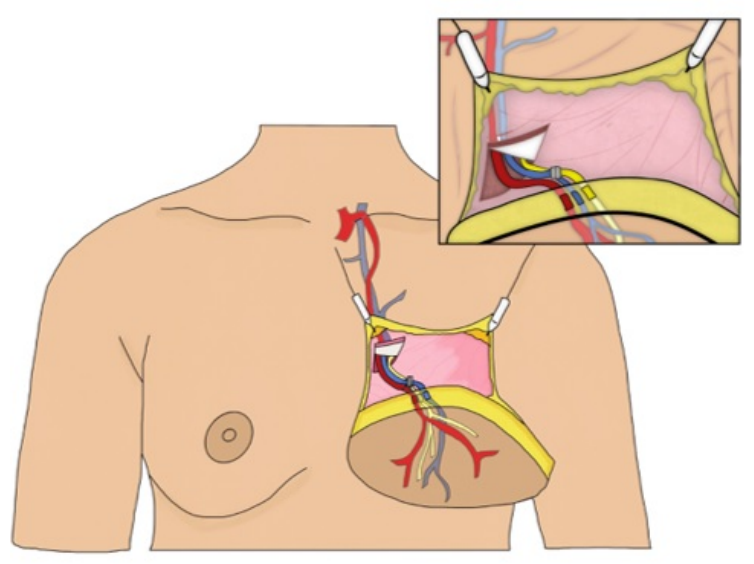

\section{Neurotización cutánea indirecta}

Se ha descrito la neurotización cutánea indirecta en un área reconstruida con injertos de piel, por medio de la invasión de nuevas fibras nerviosas desde la periferia del defecto reconstruido y desde el lecho receptor (48), aproximadamente a partir de los 40 días de su integración. La mayoría de estas fibras viajan por vainas de células de Schwann dejadas por los nervios originales o a lo largo de nuevos vasos formados durante la fase de neovascularización (49). A los dos o tres meses, dichas fibras nerviosas aumentan en número y se localizan en los folículos pilosos, las glándulas sudoríparas y los receptores sensitivos cutáneos (50), y forman una nueva red neural. La sensación de dolor se recupera primero, y aun cuando puede presentarse hipersensibilidad hasta un año después, al igual que la sensación táctil y de temperatura (51), este tipo de neurotización se ve con más frecuencia en injertos de piel de espesor total (52).

\section{Neurotización corneal}

La sensibilidad corneal está dada por la inervación desde el nervio oftálmico, de la primera división del nervio trigémino, fibras nerviosas que penetran la capa de Bowman y forman el plexo nervioso subbasal (53), esencial para la estructura y función del ojo, que permiten el reflejo palpebral y preservan el epitelio corneal (54). La anestesia corneal tiene varias causas; la principal es la queratopatía neurotrófica, secundaria a neuropatía trigémina por virus herpes, la cual lleva a cambios degenerativos de la córnea y la conjuntiva (55).

La reinervación de la córnea se puede obtener por medio de la disección y tunelización del lado contralateral de las ramas de los nervios supraorbital y supratroclear, para ser insertados alrededor del limbo esclerocorneal (56) (figura 9) o utilizando injertos de nervio sural desde el nervio supratroclear contralateral al ojo afectado (57).

\section{Figura 9}

En la técnica de neurotización corneal los nervios supraorbitario o supratroclear se transfieren al área contralateral o ipsilateral en la superficie ocular y se fijan debajo de la conjuntiva

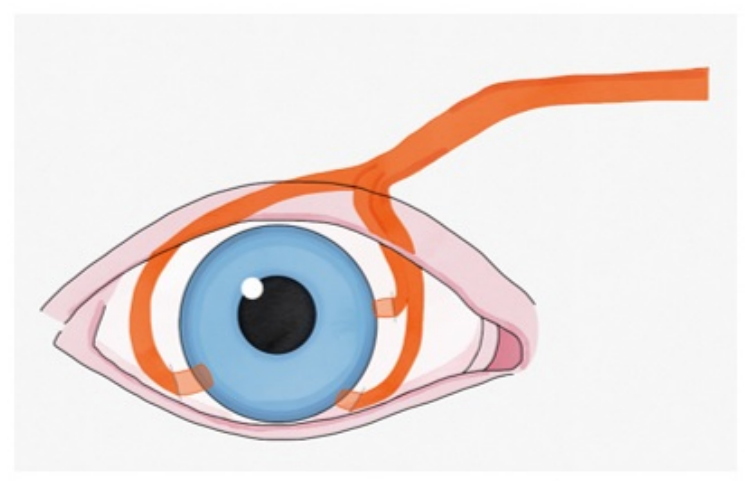

La reinervación o neurotización completa de la córnea se ha visto entre 18 y 24 meses después de este procedimiento (58).

\section{Discusión}

La neurotización es un fenómeno celular y molecular indispensable para la recuperación 
de funciones motoras y sensitivas perdidas, mediante la invasión de brotes axonales a diferentes tejidos. El más conocido es la neurotización de los tejidos de un nervio lesionado, de donde deriva su nombre.

Los diferentes tipos de neurotizaciones tienen amplias aplicaciones en una importante variedad de lesiones del sistema nervioso periférico, proceso fundamental para lograr resultados satisfactorios en los pacientes tratados.

La capacidad de cada neurona, que hace parte del nervio comprometido, de poder autorregenerarse mediante diferentes procesos moleculares permite que la neurotización se lleve a cabo, siempre y cuando existan las condiciones para que dichos eventos sucedan de forma óptima.

\section{Conclusiones}

El conocimiento de fenómenos como la neurotización permite que el personal de salud comprometido con pacientes que sufren lesiones del sistema nervioso periférico tengan más opciones de tratamiento y logren mejores resultados. Ello redunda en recuperaciones funcionales exitosas, así como incentiva el estudio y la investigación para proponer nuevas técnicas quirúrgicas o mejorar las ya existentes.

\section{Conflicto de intereses}

Los autores no declaran conflictos de intereses.

\section{Referencias}

1. Nieto L. Lesiones nerviosas de la mano. En Coiffman F. Cirugía plástica, reconstructiva y estética. 3. ${ }^{-}$ ed. Medellín: Amolca; 2008. p. 4450-7.

2. Meals RA, Nelissen RG. The origin and meaning of "neurotization". J Hand Surg Am. 1995 Jan;20(1):144-6.

3. Bentolila V, Nizard R, Bizot P, Sedel L. Complete traumatic brachial plexus palsy. Treatment and outcome after repair. J Bone Joint Surg Am. 1999 Jan;81(1):20-8. https://doi.org/10.2106 /00004623-199901000-00004

4. Boyd KU, Nimigan AS, Mackinnon SE. Nerve reconstruction in the hand and upper extremity. Clin Plast Surg. 2011 Oct;38(4):643-60. https://doi.org /10.1016/j.cps.2011.07.008

5. Ramachandran S, Midha R. Recent advances in nerve repair. Neurol India. 2019;67 (Supplement):S106-S114.

6. Lundborg G, Rosén B, Dahlin L, Danielsen N, Holmberg J. Tubular versus conventional repair of median and ulnar nerves in the human forearm: early results from a prospective, randomized, clinical study. J Hand Surg. 1997;22 (1):99-106.

7. Moore AM. Nerve transfers to restore upper extremity function: a paradigm shift. Front Neurol. 2014;5:40. https://doi.org/10.3389/fne ur.2014.00040

8. Vanaclocha-Vanaclocha V, OrtizCriado JM, Sáiz-Sapena N, Vanaclocha N. Nerve transfers in the treatment of peripheral nerve injuries. En: Colette Mauricio A, editora. Peripheral nerve regeneration: from surgery to new therapeutic approaches including biomaterials and cell-based therapies development. IntechOpen; 2017. http s://doi.org/10.5772/67948

9. Doi K. Distal nerve transfer: perspective of reconstructive microsurgery. J Reconstr Microsurg. 2018;34(9):675-7.

10. Tung TH. Nerve transfers. Clin Plast Surg. 2014;41(3):551-9.

11. Martínez F, Sevilla B, García J, López A. Técnicas de transferencia nerviosa en lesiones del miembro superior. Rev Iberoam Cir Mano. 2017;45:57-67.

12. Swanson AN, Wolfe SW, Khazzam M, Feinberg J, Ehteshami J, Doty S. 
Comparison of neurotization versus nerve repair in an animal model of chronically denervated muscle. J Hand Surg Am. 2008;33(7):1093-9.

13. Lee SK, Wolfe SW. Nerve transfers for the upper extremity: new horizons in nerve reconstruction. J Am Acad Orthop Surg. 2012;20(8):506-17.

14. Viterbo F, Amr AH, Stipp EJ, Reis FJ. End-to-side neurorrhaphy: past, present, and future. Plast Reconstr Surg. 2009;124:351-8.

15. Hayashi A, Pannucci C, Moradzadeh A, Kawamura D, Magill C, Hunter DA, et al. Axotomy or compression is required for axonal sprouting following endto-side neurorrhaphy. Exp Neurol. 2008;211(2):539-50.

16. Isaacs J, Allen D, Chen LE, Nunley J 2nd. Reverse end-to-side neurotization. J Reconstr Microsurg. 2005;21(1):43-8.

17. Kale SS, Glaus SW, Yee A, Nicoson MC, Hunter DA, Mackinnon SE, et al. Reverse end-to-side nerve transfer: from animal model to clinical use. J Hand Surg. 2011;36(10):1631-9.

18. Jandali D, Revenaugh PC. Facial reanimation: an update on nerve transfers in facial paralysis. Curr Opin Otolaryngol Head Neck Surg. 2019;27(4):231-6.

19. Jandali D, Revenaugh PC. Facial reanimation: an update on nerve transfers in facial paralysis. Curr Opin Otolaryngol Head Neck Surg. 2019;27(4):231-236.

20. Zhao X, Lao J, Hung LK, Zhang GM, Zhang LY, Gu YD. Selective neurotization of the median nerve in the arm to treat brachial plexus palsy. Surgical technique. J Bone Joint Surg Am. 2005;87 Suppl 1(1):122-35.

21. Azze RJ, Mattar Júnior J, Ferreira MC, Starck R, Canedo
AC. Extraplexual neurotization of brachial plexus. Microsurgery. 1994;15(1):28-32.

22. Chuang DC-C. Neurotization and free muscle transfer for brachial plexus avulsion injury. Hand Clin. 2007;23(1):91-104.

23. Bermúdez L, Nieto L. Massetericfacial nerve anastomosis: case report. J Reconstr Microsurg. 2004;21(1):25-30.

24. Barbour J, Yee A, Kahn LC, Mackinnon SE. Supercharged endto-side anterior interosseous to ulnar motor nerve transfer for intrinsic musculature reinnervation. J Hand Surg Am. 2012;37(10):2150-9.

25. Keles MK, Aykan A. Effect of axonal length on direct neuromuscular neurotization: an experimental study. Turk Neurosurg. 2019;29(1):110-4.

26. Konofaos P, Wallace R. Basic science of muscle neurotization: a review. J Reconstr Microsurg. 2015;31(7):481-6.

27. Brunelli G, Brunelli G. Direct muscle neurotization. J Reconstr Microsurg. 1993;9(2):81-9.

28. Brunelli G. Direct and muscular neurotisation of paralyzed muscles. J Hand Surg. 1982;7(6):572-579. https://doi.org/10. 1016/S0363-5023(82)80106-8

29. Heineke H. Die direkte Einpflanzung den Nerven in der Muskel. Centralbl F Chir. 1914;41:464-66.

30. Elsberg CA. Experiments on motor nerve regeneration and the direct neurotization of paralized muscles by their own and foreing nerves. Science. 1917;45:318-20.

31. Steindler A. The direct implantation of motor nerve upon muscle tissue (neurotization): an experimental and clinical study. J Iowa M Soc. 1915;5:436-9. 
32. Hoffman H. Fate of interrupted nerve fibres regenerating into partially denervated muscles. Aust J Exp Biol Med. 1951;29:211.

33. Birch R. Reparación nerviosa. En: Green's cirugía de la mano. Vol. 1. Madrid: Marbán; 2007.

34. Guth L, Zalewski AA. Disposition of cholinesterase following implantation of nerve into innervated and denervated muscle. Exp Neurol. 1963;7 (4):316-26.

35. Park DM, Shon SK, Kim YJ. Direct Muscle neurotization in Rat Soleus Muscle. J Reconstr Microsurg. 2000;16(5):379-84.

36. Elsberg CA. Experiments on motor nerve regenearation and the direct neurotization of paralyzed muscles by their own and by foreing nerves. Science. 1917;45(1161):318-20.

37. Cárdenas-Mejía A, Palafox D, Téllez-Palacios D, Contreras-Mérida S, Maza-Krzeptowsky L, López R, Hernández T. Levator palpebrae superioris muscle direct neurotization. J Craniofac Surg. 2017;28(8):747-8.

38. Millesi $\mathrm{H}$. Late results of the interfascicular nerve transplant: restorative surgery of lesions of the brachial plexus. Rev Med Suisse Romande. 1973;93(7):511-9.

39. Charous SJ, Hotaling JM, Burgess BD, Sappington JM, Park J, Turek G, Foecking EM. Muscle-nerve-muscle grafting for facial reanimation in rats. Ann Otol Rhinol Laryngol. 2017;126(4):261-7.

40. El-Kashlan HK, Carroll WR, Hogikyan ND, Chepeha DB, Kileny PR, Esclamado RM. Selective cricothyroid muscle reinnervation by muscle-nerve-muscle neurotization. Arch Otolaryngol Neck Surg. 2001;127(10):1211.
41. Urbanchek MG, Ganz DE, Aydin MA, van der Meulen JH, Kuzon WM. Muscle-nerve-muscle neurotization for the reinnervation of denervated somatic muscle. Neurol Res. 2004;26(4):388-94.

42. Sobotka S, Mu L. Muscle reinnervation with nerve-muscleendplate band grafting technique: correlation between force recovery and axonal regeneration. J Surg Res. 2015;195(1):144-51.

43. Kermer Ch, Millesi W, Paternostro T, Nuhr M. Muscle-nerve-muscle neurotization of the orbicularis oris muscle. J Cranio-Maxillofac Surg. 2001;29(5):302-6.

44. Hogikyan ND, Urbanchek M, Johns MM, Carroll WR, Kileny PR, Kuzon WM. motion-specific laryngeal reinnervation using muscle-nervemuscle neurotization. Ann Otol Rhinol Laryngol. 2001;110(9):801-10.

45. Taminato $\mathrm{M}$, Tomita $\mathrm{K}$, Yano K, Otani N, Kuroda K, Kubo T. Targeted sensory reinnervation by direct neurotization of skin: an experimental study in rats. J Plast Reconstr Aesthet Surg. 2021;S1748-6815(21)00035-8. https:// doi.org/10.1016/j.bjps.2020.12.101

46. Hermanson A, Dalsgaard CJ, Arnander C, Lindblom U. Sensibility and cutaneous reinnervation in free flaps. Plast Reconstr Surg. 1987;79(3):422-7.

47. Weissler JM, Koltz PF, Carney MJ, Serletti JM, Wu LC. Sifting through the evidence: a comprehensive review and analysis of neurotization in breast reconstruction. Plast Reconstr Surg. 2018;141(3):550-65.

48. Tremp M, Waldkircher NJ, Wang W, Oranges CM, di Summa PG, Zhang Y, Wang W, Schaefer DJ, Kalbermatten DF. Sensory assessment of meshed skin 
grafts over free gracilis muscle flaps without nerve coaptation for lower extremity reconstruction. Arch Plast Surg. 2021;48(2):224-30. https://doi.or g/10.5999/aps.2019.00584

49. Bayramiçli M, Jackson IT, Herschman B. Innervation of skin grafts over free muscle flaps. Br J Plast Surg. 2000;53(2):130-6.

50. Ponte B. Grafted skin: observations on innervation and other qualities. Acta Chir Scand. 1960:55-61.

51. Fitzgerald MJ, Martin F, Paletta FX. Innervation of skin grafts. Surg Gynecol Obstet. 1967;124(4):808-12.

52. Weiß-Becker C, Fruhstorfer $\mathrm{H}$, Friederich $\mathrm{H}-\mathrm{C}$, Winter $\mathrm{H}$. reinnervation of split skin grafts in humans: comparison of two different methods of operation. Scand J Plast Reconstr Surg Hand Surg. 1998;32(2):157-62.

53. Koaik M, Baig K. Corneal neurotization. Curr Opin Ophthalmol. 2019;30(4):292-8.

54. Wolkow N, Habib LA, Yoon MK, Freitag SK. Corneal neurotization: review of a new surgical approach and its developments. Semin Ophthalmol. 2019;34(7-8):473-87.

55. Kolseth CM, Charlson ES, Kossler AL. Corneal neurotization: a surgical treatment for neurotrophic keratopathy. J Neuroophthalmol. 2020;40(2):11-2.

56. Terzis J, Dryer M, Bruce B. Corneal neurotization: a novel solution to neurotrophic keratopathy. Plast Reconstr Surg. 2009;123(1):112-20. https://doi.org/10 $.1097 /$ PRS.0b013e3181904d3a

57. Bains RD, Elbaz U, Zuker RM, Ali A, Borschel GH. corneal neurotization from the supratrochlear nerve with sural nerve grafts: a minimally invasive approach. Plast Reconstr Surg. 2015;135(2):397-400.

58. Lueke JN, Holtmann C, Beseoglu K, Geerling G. Korneale neurotisation [corneal neurotization]. Ophthalmologe. 2020;117(3):248-52. 